\title{
Selected Papers from IIKII 2019 Conferences in Symmetry
}

\author{
Teen-Hang Meen ${ }^{1, * \mathbb{D}}$, Charles Tijus ${ }^{2} \mathbb{C}$ and Jih-Fu Tu ${ }^{3, *}$ \\ 1 Department of Electronic Engineering, National Formosa University, Yunlin 632, Taiwan \\ 2 Director of the Cognitions Director of the Cognitions Humaine et Artificielle Laboratory, University Paris 8, \\ Paris 93526, France; tijus@univ-paris8.fr \\ 3 Department of Industrial Engineering and Management, St. John's University, New Taipei City 25135, \\ Taiwan \\ * Correspondence: thmeen@nfu.edu.tw (T.-H.M.); tu@mail.sju.edu.tw (J.-F.T.)
}

Received: 21 April 2020; Accepted: 21 April 2020; Published: 26 April 2020

\begin{abstract}
The International Institute of Knowledge Innovation and Invention (IIKII) is an institute that promotes the exchange of innovations and inventions, and establishes a communication platform for international innovations and researches. In 2019, IIKII cooperated with the Institute of Electrical and Electronics Engineers (IEEE) Tainan Section Sensors Council to hold IEEE conferences such as IEEE ICIASE 2019, IEEE ECBIOS 2019, IEEE ICKII 2019, ICUSA-GAME 2019, and IEEE ECICE 2019. This Special Issue entitled "Selected Papers from IIKII 2019 conferences" aims to select excellent papers from IIKII 2019 conferences, including symmetry in physics, chemistry, biology, mathematics, and computer science, etc. It selected 21 excellent papers from 750 papers presented in IIKII 2019 conferences on the topic of symmetry. The main goals of this Special Issue are to encourage scientists to publish their experimental and theoretical results in as much detail as possible, and to discover new scientific knowledge relevant to the topic of symmetry.
\end{abstract}

Keywords: physics symmetry; mathematics symmetry; computer Science

\section{Introduction}

Symmetry in language refers to a sense of harmonious and beautiful proportion and balance. In mathematics, "symmetry" has a more precise definition, where an object is invariant to any of the various transformations, including reflection, rotation, or scaling. Mathematical symmetry may be observed with respect to the passage of time; as a spatial relationship; through geometric transformations; through other kinds of functional transformations; and as an aspect of abstract objects, theoretic models, and even knowledge itself. Recently, the symmetry theorem and simulation have been widely applied in engineering to improve the developments of new technologies.

In addition, the International Institute of Knowledge Innovation and Invention (IIKII, http://www.iikii.org) is an institute that promotes the exchange of innovations and inventions, and establishes a communication platform for international innovations and researches. In 2019, IIKII cooperated with the Institute of Electrical and Electronics Engineers (IEEE) Tainan Section Sensors Council to hold IEEE conferences such as IEEE ICIASE 2019, IEEE ECBIOS 2019, IEEE ICKII 2019, ICUSA-GAME 2019, and IEEE ECICE 2019. This Special Issue entitled "Selected Papers from IIKII 2019 conferences" aims to select excellent papers from IIKII 2019 conferences, including symmetry in physics, chemistry, biology, mathematics, and computer science, etc. It selected 21 excellent papers from 750 papers presented in IIKII 2019 conferences on the topic of symmetry. The main goals of this Special Issue are to encourage scientists to publish their experimental and theoretical results in as much detail as possible, and to discover new scientific knowledge relevant to the topic of symmetry. 


\section{The Topic of Symmetry}

This special issue selected 21 excellent papers from 750 papers presented in IIKII 2019 conferences on the topic of symmetry. The published papers are introduced as follows: Kubí cek et al. reported "Proposal of Technological Geographic Information System (GIS) Support as Part of Resident Parking in Large Cities-Case Study, City of Brno" [1]. The aim of this study is to design and optimize the integrated collection of image data localized by satellite Global Satellite Navigation Systems (GNSS) technologies in the GIS environment to support the resident parking system, including an evaluation of its effectiveness. To achieve this goal, a residential parking monitoring system was designed and implemented, based on dynamic monitoring of the parking state using a vehicle equipped with a digital camera system and Global Satellite Navigation Systems (GNSS) technology for measuring the vehicle position, controlled by spatial and attribute data flow from static and dynamic spatial databases in the Geographic Information System (GIS), which integrate the whole monitoring system. The control algorithm of a vehicle passing through the street network works on the basis of graph theory with a defined recurrence interval for the same route, taking into account other parameters such as the throughput of the street network at a given time, its traffic signs, and the usual level of traffic density. Statistics after one year of operation show that the proposed system significantly increased the economic yield from parking areas from the original $30 \%$ to $90 \%$ and reduced the overall violation of parking rules to only $10 \%$. It further increased turnover and, thus, the possibility of short-term parking for visitors, and it also ensured availability of parking for residents in the historical center of Brno and surrounding monitored areas.

Lee et al. reported the "Design and Implementation of Virtual Private Storage Framework Using Internet of Things Local Networks" [2]. This paper presents a virtual private storage framework (VPSF) using Internet of Things (IoT) local networks. The VPSF uses the extra storage space of sensor devices in an IoT local network to store users' private data, while guaranteeing expected network lifetime, by partitioning the storage space of a sensor device into data and system volumes and, if necessary, logically integrating the extra data volumes of the multiple sensor devices to virtually build a single storage space. When user data need to be stored, the VPSF gateway divides the original data into several blocks and selects the sensor devices in which the blocks will be stored based on their residual energy. The blocks are transmitted to the selected devices using the modified speedy block-wise transfer (BlockS) option of the constrained application protocol (CoAP), which reduces communication overhead by retransmitting lost blocks without a retransmission request message. To verify the feasibility of the VPSF, an experimental implementation was conducted using the open-source software libcoap. The results demonstrate that the VPSF is an energy-efficient solution for virtual private storage because it averages the residual energy amounts for sensor devices within an IoT local network and reduces their communication overhead.

Kwon et al. reported "Failure Prediction Model Using Iterative Feature Selection for Industrial Internet of Thing" [3]. This paper presents a failure prediction model using iterative feature selection, which aims to accurately predict the failure occurrences in industrial Internet of Things (IIoT) environments. In general, vast amounts of data are collected from various sensors in an IIoT environment, and they are analyzed to prevent failures by predicting their occurrence. However, the collected data may include data irrelevant to failures and thereby decrease the prediction accuracy. To address this problem, the authors propose a failure prediction model using iterative feature selection. To build the model, the relevancy between each feature (i.e., each sensor) and the failure was analyzed using the random forest algorithm, to obtain the importance of the features. Then, feature selection and model building were conducted iteratively. In each iteration, a new feature was selected considering the importance and added to the selected feature set. The failure prediction model was built for each iteration via the support vector machine (SVM). Finally, the failure prediction model having the highest prediction accuracy was selected. The experimental implementation was conducted using open-source $R$. The results showed that the proposed failure prediction model achieved high prediction accuracy. 
Lan et al. reported "Symmetric Modeling of Communication Effectiveness and Satisfaction for Communication Software on Job Performance" [4]. Users in the Taiwanese community send messages or share information through communication software that leads to more dependence from business. Various business problems have been solved and job performance has increased through the diversified functions on communication software. Thus, this research supposed that staff are willing to continuously use communication software LINE (a new communication app that allows one to make FREE voice calls and send FREE messages), and they agree that the varied functions of the communication software would mean that information delivery more symmetrically affects their job performance. According to the research outcomes, communication effectiveness significantly influenced communication satisfaction and job performance, and communication satisfaction significantly influenced job performance. As organizational communication must be conducted through media that disseminate information, and different media have different communication effects, the relationship between communication effectiveness and job performance was completely mediated by communication satisfaction. The research suggested companies or organizations use LINE as a symmetric communication method to not only help employees improve their job performance, but also help enterprises achieve their goals or raise their profit, or even steady development for enterprises.

Li et al. reported "Homomorphic Encryption-Based Robust Reversible Watermarking for 3D Model" [5]. Robust reversible watermarking in an encrypted domain is a technique that preserves privacy and protects copyright for multimedia transmission in the cloud. In general, most models of buildings and medical organs are constructed by three-dimensional (3D) models. A 3D model shared through the internet can be easily modified by an unauthorized user, and in order to protect the security of 3D models, a robust reversible 3D models watermarking method based on homomorphic encryption is necessary. In this study, a 3D model is divided into non-overlapping patches, and the vertex in each patch is encrypted by using the Paillier cryptosystem. On the cloud side, in order to utilize the addition and multiplication homomorphism of the Paillier cryptosystem, three direction values of each patch are computed for constructing the corresponding histogram, which is shifted to embed the watermark. For obtaining watermarking robustness, the robust interval is designed in the process of histogram shifting. The watermark can be extracted from the symmetrical direction histogram, and the original encrypted model can be restored by histogram shifting. Moreover, the process of watermark embedding and extraction are symmetric. Experimental results show that compared to the existing watermarking methods in encrypted 3D models, the quality of the decrypted model is improved. Moreover, the proposed method is robust to common attacks, such as translation, scaling, and Gaussian noise.

Zhang et al. reported "A Matching Pursuit Algorithm for Backtracking Regularization Based on Energy Sorting" [6]. This paper proposes a matching pursuit algorithm for backtracking regularization based on energy sorting. This algorithm uses energy sorting for secondary atom screening to delete individual wrong atoms through the regularized orthogonal matching pursuit (ROMP) algorithm backtracking. The support set is continuously updated and expanded during each iteration. While the signal energy distribution is not uniform, or the energy distribution is in an extreme state, the reconstructive performance of the ROMP algorithm becomes unstable if the maximum energy is still taken as the selection criterion. The proposed method for the regularized orthogonal matching pursuit algorithm can be adopted to improve those drawbacks in signal reconstruction due to its high reconstruction efficiency. The experimental results show that the algorithm has a proper reconstruction.

Ye et al. reported "Incorporating Particle Swarm Optimization into Improved Bacterial Foraging Optimization Algorithm Applied to Classify Imbalanced Data" [7]. In this paper, particle swarm optimization is incorporated into an improved bacterial foraging optimization algorithm, which is applied to classify imbalanced data to solve the problem of how original bacterial foraging optimization easily falls into local optimization. In this study, the borderline synthetic minority oversampling technique (Borderline-SMOTE) and Tomek link are used to pre-process imbalanced data. 
Then, the proposed algorithm is used to classify the imbalanced data. In the proposed algorithm, the chemotaxis process is first improved. The particle swarm optimization (PSO) algorithm is used to search first and then treat the result as bacteria, improving the global searching ability of bacterial foraging optimization (BFO). Secondly, the reproduction operation is improved and the selection standard of survival of the cost is improved. Finally, the authors improve elimination and dispersal operation, and the population evolution factor is introduced to prevent the population from stagnating and falling into a local optimum. In this paper, three data sets are used to test the performance of the proposed algorithm. The simulation results show that the classification accuracy of the proposed algorithm is better than the existing approaches.

Lin et al. reported "Application of Gray Relational Analysis and Computational Fluid Dynamics to the Statistical Techniques of Product Designs" [8]. During the development of fan products, designers often encounter gray areas when creating new designs. Without clear design goals, development efficiency is usually reduced, and fans are the best solution for studying symmetry or asymmetry. Therefore, fan designers need to figure out an optimization approach that can simplify the fan development process and reduce associated costs. This study provides a new statistical approach using gray relational analysis (GRA) to analyze and optimize the parameters of a particular fan design. During the research, it was found that the single fan uses an asymmetry concept with a single blade as the design, while the operation of double fans is a symmetry concept. The results indicated that the proposed mechanical operations could enhance the variety of product designs and reduce costs. Moreover, this approach can relieve designers from unnecessary effort during the development process and also effectively reduce the product development time.

Hung et al. reported "Applying Educational Data Mining to Explore Students' Learning Patterns in the Flipped Learning Approach for Coding Education" [9]. In this study, the authors applied educational data mining to explore the learning behaviors in data generated by students in a blended learning course. The experimental data were collected from two classes of Python programming-related courses for first-year students in a university in northern Taiwan. During the semester, high-risk learners could be predicted accurately by data generated from the blended educational environment. The f1-score of the random forest model was 0.83 , which was higher than the f1-score of logistic regression and decision tree. The model built in this study could be extrapolated to other courses to predict students' learning performance, where the F1-score was 0.77 . Furthermore, the authors used machine learning and symmetry-based learning algorithms to explore learning behaviors. By using the hierarchical clustering heat map, this study could define the students' learning patterns including the positive interactive group, stable learning group, positive teaching material group, and negative learning group. These groups also corresponded to the student conscious questionnaire. With the results of this research, teachers can use the mid-term forecasting system to find high-risk groups during the semester and remedy their learning behaviors in the future.

Bartonek et al. reported "Problems of Creation and Usage of 3D Model of Structures and Theirs Possible Solution" [10]. This paper describes problems that occur when creating three-dimensional (3D) building models. The first problem is geometric accuracy; the next is the quality of visualization of the resulting model. The main cause of this situation is that current computer-aided design (CAD) software does not have the sufficient means to precision mapping the measured data of a given object in the field. Therefore, the process of 3D model creation is mainly a relatively high proportion of manual work when connecting individual points, approximating curves and surfaces, or laying textures on surfaces. In some cases, it is necessary to generalize the model in the CAD system, which degrades the accuracy and quality of field data. The paper analyzes these problems and then recommends several variants for their solution. There are two basic methods described: Using topological codes in the list of coordinate points and creating new special CAD features while using Python scripts. These problems are demonstrated on examples of 3D models in practice. These are mainly historical buildings in different locations and different designs (brick or wooden structures). These are four sacral buildings in the Czech Republic (CR): The church of saints Johns of Brno-Bystrc, the Church of St. Paraskiva in 
Blansko, the Strejc's Church in Židlochovice, and the Church of St. Peter in Alcantara in Karviná city. All of the buildings were geodetically surveyed by the terrestrial method while using the total station. The 3D model was created in both cases in the program AUTOCAD v. 18 and MicroStation.

Chen et al. reported "A Balance Interface Design and Instant Image-based Traffic Assistant Agent Based on GPS and Linked Open Data Technology" [11]. This paper aims to integrate government open data and global positioning system (GPS) technology to build an instant image-based traffic assistant agent with user-friendly interfaces, thus providing more convenient real-time traffic information for users and relevant government units. The proposed system is expected to overcome the difficulty of accurately distinguishing traffic information and to solve the problem of some road sections not providing instant information. Taking the New Taipei City Government traffic open data as an example, the proposed system can display information pages at an optimal size on smartphones and other computer devices, and integrate database analysis to instantly view traffic information. Users can enter the system without downloading the application and can access the cross-platform services using device browsers. The proposed system also provides a user reporting mechanism, which informs vehicle drivers on congested road sections about road conditions. Comparison and analysis of the system with similar applications show that although they have similar functions, the proposed system offers more practicability, better information accessibility, excellent user experience, and an approximately optimal balance (a kind of symmetry) of the important items of the interface design.

Fan et al. reported "Investigation of High-Efficiency Iterative ILU Preconditioner Algorithm for Partial-Differential Equation Systems" [12]. In this paper, the authors investigate an iterative incomplete lower and upper (ILU) factorization preconditioner for partial-differential equation systems. The authors discretize the partial-differential equations into linear equation systems. An iterative scheme of linear systems is used. ILU preconditioners of linear systems are performed on the different computation nodes of multi-central processing unit (CPU) cores. First, the preconditioner of general tridiagonal matrix equations is tested on supercomputers. Then, the effects of partial-differential equation systems on the speedup of parallel multiprocessors are examined. The numerical results estimate that the parallel efficiency is higher than in other algorithms.

Chuang et al. reported "Parameter Optimization for Computer Numerical Controlled Machining Using Fuzzy and Game Theory" [13]. In this study, the precision computerized numerically controlled (CNC) cutting process was chosen as an example, while tool wear and cutting noise were chosen as the research objectives of CNC cutting quality. The effects of quality optimization were verified using the depth of cut, cutting speed, feed rate, and tool nose runoff as control parameters, and actual cutting on a CNC lathe was performed. Further, the relationships between Fuzzy theory and control parameters, as well as quality objectives, were used to define semantic rules to perform fuzzy quantification. The quantified output value was introduced into game theory to carry out the multi-quality bargaining game. Through the statistics of strategic probability, the strategy with the highest total probability was selected to obtain the optimum plan of multi-quality and multi-strategy. Under the multi-quality optimum parameter combination, the tool wear and cutting noise, compared to the parameter combination recommended by the cutting manual, was reduced by $23 \%$ and $1 \%$, respectively. This research can indeed ameliorate the multi-quality cutting problem. The results of the research provided the technicians with a set of all-purpose economic prospective parameter analysis methods in the manufacturing process to enhance the international competitiveness of the automated CNC industry.

Bao et al. reported "An Efficient Data Transmission with GSM-MPAPM Modulation for an Indoor VLC System" [14]. The objective of this study was to put forward an efficient and theoretical scheme that is based on generalized spatial modulation to reduce the bit error ratio in indoor short-distance visible light communication. The scheme was implemented while using two steps in parallel: (1) The multi-pulse amplitude and the position modulation signal were generated by combining multi-pulse amplitude modulation with multi-pulse position modulation using transmitted information, and (2) certain light-emitting diodes were activated by employing the idea of generalized 
spatial modulation to convey the generated multi-pulse amplitude and position modulation optical signals. Furthermore, pulse width modulation was introduced to achieve dimming control in order to improve the anti-interference ability to the ambient light of the system. The two steps above involved the information theory of communication. An embedded hardware system, which was based on the C8051F330 microcomputer and included a transmitter and a receiver, was designed to verify the performance of this new scheme. Subsequently, the verifiability experiment was carried out. The results of this experiment demonstrated that the proposed theoretical scheme of transmission was feasible and could lower the bit error ratio (BER) in indoor short-distance visible light communication while guaranteeing indoor light quality.

Hsueh et al. reported "Fault Diagnosis System for Induction Motors by CNN Using Empirical Wavelet Transform" [15]. In this paper, a novel methodology is demonstrated to detect the working condition of a three-phase induction motor and classify it as a faulty or healthy motor. The electrical current signal data are collected for five different types of fault and one normal operating condition of the induction motors. The first part of the methodology illustrates a pattern recognition technique based on the empirical wavelet transform, to transform the raw current signal into two-dimensional (2-D) grayscale images comprising the information related to the faults. Second, a deep convolutional neural network (CNN) model is proposed to automatically extract robust features from the grayscale images to diagnose the faults in the induction motors. The experimental results show that the proposed methodology achieves a competitive accuracy in the fault diagnosis of the induction motors and that it outperformed the traditional statistical and other deep learning methods.

Shieh et al. reported "Forecasting for Ultra-Short-Term Electric Power Load Based on Integrated Artificial Neural Networks" [16]. In order to improve power production efficiency, an integrated solution regarding the issue of electric power load forecasting was proposed in this study. The solution proposed was to, in combination with persistence and search algorithms, establish a new integrated ultra-short-term electric power load forecasting method based on the adaptive-network-based fuzzy inference system (ANFIS) and back-propagation neural network (BPN), which can be applied in forecasting electric power load in Taiwan. The research methodology used in this paper was mainly to acquire and process the all-day electric power load data of Taiwan Power and execute preliminary forecasting values of the electric power load by applying ANFIS, BPN, and persistence. The preliminary forecasting values of the electric power load obtained therefrom were called suboptimal solutions and the optimal weighted value was finally determined by applying a search algorithm through integrating the above three methods by weighting. In this paper, the optimal electric power load value was forecasted based on the weighted value obtained therefrom. It was proven through experimental results that the solution proposed in this paper can be used to accurately forecast electric power load, with minimal error.

Wang et al. reported "Behavior Modality of Internet Technology on Reliability Analysis and Trust Perception for International Purchase Behavior" [17]. The main research question that this study intends to answer is, "What do users do when a YouTube advertisement appears? Do they avoid or confront them?" The aim of this study is to explore the perceptions and related behaviors of international purchasing and consumers' trust of YouTube advertisements. Statistical analyses focus on the demographics of a sample population in Thailand. The findings are based on data obtained by a questionnaire, the results of which were analyzed by $\mathrm{t}$-test and multiple regression. The results indicate that YouTube advertising has a significant effect on behavioral trends. Moreover, the subjects in the sample reported that they are more likely to avoid YouTube ads than confront them. The study subjects have a low satisfaction with YouTube advertising, and males have a significantly lower satisfaction than females. This study also analyzes the reliability of trust perception toward purchasing. The results indicate that the reliability is greater than $90 \%$ at an $\alpha$ level of $5 \%$ and a $95 \%$ confidence interval.

Lin et al. reported "Application of the Symmetric Model to the Design Optimization of Fan Outlet Grills" [18]. In this study, different designs of the opening pattern of computer fan grills were investigated. The objective of this study was to propose a simulation analysis and compare it to the 
experimental results for a set of optimized fan designs. The FLUENT computational fluid dynamics (CFD) simulation software was used to analyze the fan blade flow. The experimental results obtained by the simulation analysis of the optimized fan designs were analyzed and compared. The effect of different opening pattern designs on the resulting airflow rate was investigated. Six types of fans with different grills were analyzed. The airflow velocity distribution in the simulated flow channel indicated that the wind speed efficiency of the fan and its influence were comparable to the experimental model. The air was forced by the fan into the air duct. The flow path was separately measured by analog instruments. The three-dimensional flow field was determined by performing a wind speed comparison on nine planes containing the mainstream velocity vector. Moreover, the three-dimensional curved surface flow field at the outlet position and the highest fan rotation speed were investigated. The air velocity distribution at the inlet and the outlet of the fan indicated that among the air outlet opening designs, the honeycomb-shaped air outlet displayed the optimal performance by investigating the fan characteristics and the estimated wind speed efficiency. These optimized designs were the most ideal configurations to compare these results. The air flow rate was evenly distributed at the fan inlet.

Chen et al. reported "Energy Consumption Load Forecasting Using a Level-Based Random Forest Classifier" [19]. In this study, a conventional method of level prediction with a pattern recognition approach was performed by first predicting the actual numerical values using typical pattern-based regression models, and then classifying them into pattern levels (e.g., low, average, and high). A proposed prediction with a pattern recognition scheme was developed to directly predict the desired levels using simpler classifier models without undergoing regression. The proposed pattern recognition classifier was compared to its regression method using a similar algorithm applied to a real-world energy dataset. A random forest (RF) algorithm, which outperformed other widely used machine learning (ML) techniques in previous research, was used in both methods. Both schemes used similar parameters for training and testing simulations. After 10 cross-training validations and five averaged repeated runs with random permutations per data splitting, the proposed classifier shows better computation speed and higher classification accuracy than the conventional method. However, when the number of its desired levels increases, its prediction accuracy seems to decrease and approaches the accuracy of the conventional method. The developed energy level prediction, which is computationally inexpensive and has a good classification performance, can serve as an alternative forecasting scheme.

Chen et al. reported "The Computer Course Correlation between Learning Satisfaction and Learning Effectiveness of Vocational College in Taiwan" [20]. In this paper, the authors surveyed the influence of learning effectiveness in a computer course under the factors of learning attitude and learning problems for students in senior-high school. The authors followed the formula for a regression line as $\mathrm{R}=\mathrm{A}+\mathrm{BX}+\varepsilon$ and simulated it on a Statistical Product and Service Solutions (SPSS) platform with symmetry to obtain the results as follows: (1) In learning attitude, both the cognitive-level and behavior-level are positively correlated with satisfaction. This means the students have a cognitive-level and behavior-level more positively correlated with satisfaction in computer subjects and have a high degree of self-learning effectiveness. (2) In learning problems, the female students had a higher learning effectiveness than male students, and the students who practiced on the computer on their own initiative long-term each week had a higher learning effectiveness.

Gao et al. reported "Locality Sensitive Discriminative Unsupervised Dimensionality Reduction" [21]. Graph-based embedding methods receive much attention due to the use of graph and manifold information. However, conventional graph-based embedding methods may not always be effective if the data have high dimensions and have complex distributions. First, the similarity matrix only considers local distance measurement in the original space, which cannot reflect a wide variety of data structures. Second, the separation of graph construction and dimensionality reduction leads to the similarity matrix not being fully relied on because the original data usually contain lots of noise samples and features. In this paper, the authors address these problems by constructing two adjacency graphs to stand for the original structure featuring similarity and diversity of the data, and then impose 
a rank constraint on the corresponding Laplacian matrix to build a novel adaptive graph learning method, namely locality sensitive discriminative unsupervised dimensionality reduction (LSDUDR). As a result, the learned graph shows a clear block diagonal structure so that the clustering structure of data can be preserved. Experimental results on synthetic datasets and real-world benchmark data sets demonstrate the effectiveness of our approach.

Author Contributions: Writing and reviewing all papers, T.-H.M.; English editing, C.T.; Checking and correcting manuscript, J.-F.T. All authors have read and agreed to the published version of the manuscript.

Funding: This research received no external funding.

Acknowledgments: The guest editors would like to thank the authors for their contributions to this special issue and all the reviewers for their constructive reviews. We are also grateful to Dalia Su, the Managing Editor of Symmetry, for her time and efforts on the publication of this special issue for Symmetry.

Conflicts of Interest: The authors declare no conflicts of interest.

\section{References}

1. Kubíček, P.; Bartoněk, D.; Bureš, J.; Švábenský, O. Proposal of Technological GIS Support as Part of Resident Parking in Large Cities-Case Study, City of Brno. Symmetry 2020, 12, 542. [CrossRef]

2. Lee, H.-H.; Kwon, J.-H.; Kim, E.-J. Design and Implementation of Virtual Private Storage Framework Using Internet of Things Local Networks. Symmetry 2020, 12, 489. [CrossRef]

3. Kwon, J.-H.; Kim, E.-J. Failure Prediction Model Using Iterative Feature Selection for Industrial Internet of Things. Symmetry 2020, 12, 454. [CrossRef]

4. Lan, T.-S.; Chuang, K.-C.; Li, H.-X.; Tu, J.-F.; Huang, H.-S. Symmetric Modeling of Communication Effectiveness and Satisfaction for Communication Software on Job Performance. Symmetry 2020, 12, 418. [CrossRef]

5. Li, L.; Wang, S.; Zhang, S.; Luo, T.; Chang, C.-C. Homomorphic Encryption-Based Robust Reversible Watermarking for 3D Model. Symmetry 2020, 12, 347. [CrossRef]

6. Zhang, H.; Xiao, S.; Zhou, P. A Matching Pursuit Algorithm for Backtracking Regularization Based on Energy Sorting. Symmetry 2020, 12, 231. [CrossRef]

7. Ye, F.-L.; Lee, C.-Y.; Lee, Z.-J.; Huang, J.-Q.; Tu, J.-F. Incorporating Particle Swarm Optimization into Improved Bacterial Foraging Optimization Algorithm Applied to Classify Imbalanced Data. Symmetry 2020, 12, 229. [CrossRef]

8. Lin, H.-H.; Cheng, J.-H.; Chen, C.-H. Application of Gray Relational Analysis and Computational Fluid Dynamics to the Statistical Techniques of Product Designs. Symmetry 2020, 12, 227. [CrossRef]

9. Hung, H.-C.; Liu, I.-F.; Liang, C.-T.; Su, Y.-S. Applying Educational Data Mining to Explore Students' Learning Patterns in the Flipped Learning Approach for Coding Education. Symmetry 2020, 12, 213. [CrossRef]

10. Bartoněk, D.; Buday, M. Problems of Creation and Usage of 3D Model of Structures and Theirs Possible Solution. Symmetry 2020, 12, 181. [CrossRef]

11. Chen, F.-H.; Yang, S.-Y. A Balance Interface Design and Instant Image-based Traffic Assistant Agent Based on GPS and Linked Open Data Technology. Symmetry 2019, 12, 1. [CrossRef]

12. Fan, Y.-H.; Wang, L.-H.; Jia, Y.; Li, X.-G.; Yang, X.-X.; Chen, C.-C. Investigation of High-Efficiency Iterative ILU Preconditioner Algorithm for Partial-Differential Equation Systems. Symmetry 2019, 11, 1461. [CrossRef]

13. Chuang, K.-C.; Lan, T.-S.; Zhang, L.; Chen, Y.-M.; Dai, X.-J. Parameter Optimization for Computer Numerical Controlled Machining Using Fuzzy and Game Theory. Symmetry 2019, 11, 1450. [CrossRef]

14. Bao, J.-J.; Hsu, C.-L.; Tu, J.-F. An Efficient Data Transmission with GSM-MPAPM Modulation for an Indoor VLC System. Symmetry 2019, 11, 1232. [CrossRef]

15. Hsueh, Y.-M.; Ittangihal, V.R.; Wu, W.-B.; Chang, H.-C.; Kuo, C.-C. Fault Diagnosis System for Induction Motors by CNN Using Empirical Wavelet Transform. Symmetry 2019, 11, 1212. [CrossRef]

16. Shieh, H.-L.; Chen, F.-H. Forecasting for Ultra-Short-Term Electric Power Load Based on Integrated Artificial Neural Networks. Symmetry 2019, 11, 1063. [CrossRef]

17. Wang, S.-L.; Hou, Y.-T.; Kankham, S. Behavior Modality of Internet Technology on Reliability Analysis and Trust Perception for International Purchase Behavior. Symmetry 2019, 11, 989. [CrossRef] 
18. Lin, H.-H.; Cheng, J.-H. Application of the Symmetric Model to the Design Optimization of Fan Outlet Grills. Symmetry 2019, 11, 959. [CrossRef]

19. Chen, Y.-T.; Piedad, E., Jr.; Kuo, C.-C. Energy Consumption Load Forecasting Using a Level-Based Random Forest Classifier. Symmetry 2019, 11, 956. [CrossRef]

20. Chen, R.-Y.; Tu, J.-F. The Computer Course Correlation between Learning Satisfaction and Learning Effectiveness of Vocational College in Taiwan. Symmetry 2019, 11, 822. [CrossRef]

21. Gao, Y.-L.; Luo, S.-Z.; Wang, Z.-H.; Chen, C.-C.; Pan, J.-Y. Locality Sensitive Discriminative Unsupervised Dimensionality Reduction. Symmetry 2019, 11, 1036. [CrossRef] 